\title{
Veränderungsmessung und Rückfälligkeit im Risikoorientierten Sanktionenvollzug
}

\author{
Daniel Treuthardt ${ }^{1,3} \cdot$ Melanie Kröger ${ }^{2}$ \\ Eingegangen: 29. Januar 2021 / Angenommen: 17. September 2021 / Online publiziert: 29. Oktober 2021 \\ (c) Der/die Autor(en) 2021
}

\section{Zusammenfassung}

Mit der Einführung des Risikoorientierten Sanktionenvollzugs (ROS) in der Schweiz sollen Rückfälle größtmöglich reduziert und die Ressourcen der Straftäter*innen gestärkt werden. Um diesem Anspruch gerecht zu werden, wird ROS fortlaufend evaluiert und weiterentwickelt. Nun soll eine konzipierte Veränderungsmessung überprüft werden, die ROS erweitern und die Abbildung von Veränderungen transparenter und nachvollziehbarer machen könnte. Neben den demografischen und Rückfalldaten werden diverse personen- und umweltbezogene Aspekte herangezogen. Konkret wird anhand von 302 Fällen betrachtet, ob im Verlauf der Sanktion eine Verbesserung der personen- und umweltbezogenen Aspekte stattgefunden hat. Anschließend wird analysiert, ob Verbesserungen der personen- und umweltbezogenen Variablen $\mathrm{Zu}$ sammenhänge zur Rückfälligkeit aufweisen. Ebenso wird betrachtet, ob eine gute Passung von forensischem Bedarf und juristischen Rahmenbedingungen mit den Variablen sowie der Rückfälligkeit zusammenhängt. Des Weiteren wird anhand von Vergleichsdaten aus einer Stichprobe vor der Einführung von ROS die Wirksamkeit von ROS in Bezug auf Rückfälligkeit untersucht. Die untersuchten Bereiche weisen teilweise signifikante Ergebnisse auf. So deuten die Befunde darauf hin, dass die Veränderungsmessung grundsätzlich eine sinnvolle Ergänzung im ROS-Prozess darstellt. Außerdem kann die Wirksamkeit von ROS bestätigt werden. Abschließend werden Limitationen diskutiert und ein Ausblick gegeben.

Schlüsselwörter Straftäterbehandlung $\cdot$ Wirksamkeit $\cdot$ Risikominderung $\cdot$ Ressourcenstärkung $\cdot$ Wiedereingliederung

\section{Measuring change and recidivism in the risk-oriented enforcement of sanctions}

\begin{abstract}
The risk-oriented enforcement of sanctions (ROS) was introduced to reduce the risk of reoffending as well as to strengthen offender resources. To meet these goals, ROS is constantly being evaluated and enhanced. Now a change measurement is evaluated, which might further strengthen ROS as well as make changes in offenders more transparent and traceable. Demographic data, recidivism rates and different environmental and personality variables were used in an analysis of 302 cases. Offender changes in environmental and personality aspects were dissected. Furthermore, the relationship between the environmental and personality variables and recidivism was examined. Another investigated aspect was the fit between forensic needs and the legal framework. The relationship between this fit and the environmental and personality aspects as well as recidivism was analyzed as well. Furthermore, the effectiveness of ROS was measured by comparing recidivism rates pre-ROS and post-ROS introduction. Most aspects were at least partially significant. The change measurement seems to be a useful addition to the ROS. Additionally, ROS seems to be effective. Finally, limitations and an outlook are presented.
\end{abstract}

Keywords Offender treatment $\cdot$ Effectiveness $\cdot$ Risk reduction $\cdot$ Resource strengthening $\cdot$ Reintegration

Daniel Treuthardt, lic. phil.

daniel.treuthardt@ji.zh.ch

1 Bewährungs- und Vollzugsdienste, ROS-Administration, Justizvollzug und Wiedereingliederung des Kantons Zürich, Hohlstr. 552, 8090 Zürich, Schweiz
2 Fachbereich Psychologie, Universität Konstanz, 78457 Konstanz, Deutschland

3 University of Zurich, Zürich, Schweiz 


\section{Einleitung}

Als Reaktion auf unterschiedliche Rückfalltaten von Gewalt- und Sexualstraftätern wurde, beginnend mit einem Modellversuch in den Jahren 2010-2013, in den Kantonen der Ost-, West- und Innerschweiz bis 2018 das Konzept Risikoorientierter Sanktionenvollzug (ROS) eingeführt. Basierend auf dem Risk-Need-Responsivity-Prinzip nach Andrews und Bonta (2010) hat ROS zum Ziel, Rückfälle größtmöglich zu reduzieren, die Ressourcen der Straftäter*innen zu stärken und damit eine nachhaltige Resozialisierung zu fördern.

Um die Ziele bestmöglich umzusetzen, durchläuft jeder Fall im ROS 4 Prozessschritte: Triage, Abklärung, Planung und Verlauf. In der Triage wird anhand des Fall-ScreeningTools (FaST) der weitere Abklärungsbedarf festgestellt (für vertiefte Informationen zum FaST: Treuthardt und Kröger 2019). Entsprechend dem Resultat der Triage werden dann in den Schritten Abklärung und Planung mithilfe strukturierter Hilfsmittel der Veränderungsbedarf und das weitere Vorgehen zur Rückfallreduzierung und Ressourcenstärkung geplant. Im Verlauf erfolgen standardisierte Rückmeldungen durch die beteiligten Arbeitspartner (Vollzugseinrichtungen, Therapierende, Betreuende und andere Beteiligte). Für einen vertieften Überblick über ROS: LoeweBaur (2017) und Treuthardt et al. (2018).

Seit der Konzipierung wurde ROS mehrfach evaluiert und überarbeitet (Ruflin et al. 2013; Schwarzenegger et al. 2013; Loewe-Baur 2017; Treuthardt und Kröger 2019). Bislang wurden insbesondere bereits bestehende Bestandteile evaluiert und überarbeitet. Im Folgenden soll nun mit der Evaluation einer Veränderungsmessung ein Schritt dahin unternommen werden, Veränderungsmessung standardisierter in den Prozess zu implementieren. Aufgrund der großen Menge und Komplexität der dabei berücksichtigten Variablen wurde die Konzipierung der Veränderungsmessung bereits in einem eigenständigen Artikel ausführlich dargestellt (Treuthardt und Kröger 2021). Im Folgenden werden die Aspekte zur besseren Übersicht kurz aufgegriffen und im Abschn. „Methode“ weiter ausgeführt. Zudem werden Erkenntnisse zu den Variablen aus einer vorab durchgeführten Erhebung der Interrater-Reliabilität ergänzt.

\section{Personenbezogene Veränderung}

Die Veränderung der personenbezogenen Denk- und Verhaltensmuster setzt sich aus folgenden Komponenten zusammen: Veränderungspotenzial, delinquenzbezogene Prozessvariablen und Stufen der Verhaltensänderung (,stages of change" [SoC]). Das Veränderungspotenzial (bestehend aus Veränderungsfähigkeit und Veränderungsmotivation) und die daran angelehnten delinquenzbezogenen Prozessvariablen fragen nach den Ebenen Wollen (motiva- tionale Aspekte), Wissen (kognitive Aspekte) und Können (Handlungskompetenzen) in Bezug auf die deliktpräventive Bearbeitung des spezifischen Problemprofils der Person. Die SoC sind angelehnt an das Transtheoretische Modell der Verhaltensänderung nach Prochaska und DiClemente $(1984,1986)$ und die daraus resultierte Violence Risk Scale nach Haubner-MacLean et al. (2013). Laut dem Transtheoretischen Modell erfolgt Verhaltensänderung stufenförmig. Auf der ersten Stufe, Absichtslosigkeit, ist kein Problembewusstsein oder keine Absicht zur Verhaltensänderung vorhanden. Gelangt eine Person auf die nächste Stufe, $A b$ sichtsbildung, ist ein Problembewusstsein vorhanden, und erste Überlegungen werden angestellt, wie Verhalten und Umwelt verändert werden könnten. Laut der Faktorenanalyse des URICA (DiClemente und Hughes 1990) - eines gängigen Selbstbeurteilungsfragebogens zur Messung der SoC - wird die dritte Stufe Vorbereitung nicht berücksichtigt. Dementsprechend ist die nächste Stufe Handlung, auf der aktiv am Verhalten gearbeitet wird. Die Veränderungen sind jedoch instabil. Erst auf der nächsten Stufe, Aufrechterhaltung, ist ein nachhaltiges Veränderungsstreben erkennbar.

\section{Umweltbezogene Veränderung}

Die umweltbezogene Veränderung bezieht sich ebenfalls auf die Ebenen Wollen, Wissen und Können. Auch hier werden die Items auf einer 4-stufigen Likert-Skala eingeschätzt. Es werden zentrale Lebensbereiche betrachtet, in denen Probleme auftauchen, wie beispielsweise Arbeit, Finanzen, Partnerschaft und Familie oder sonstiges soziales Umfeld.

\section{Ausprägung des umwelt- und personenbezogenen Problemprofils}

Die Ausprägung betrachtet übergeordnet, wie gravierend das umwelt- und personenbezogene Problemprofil sich auf das Leben der Person auswirkt. Hierbei wird nicht nur deliktspezifisch auf die Rückfallgefahr geachtet, sondern auch die Auswirkung auf das alltägliche Leben miteinbezogen. Mit der Ausprägung wird das Ausmaß der Beeinträchtigung des psychosozialen Funktionsniveaus in verschiedenen Lebensbereichen bezeichnet, welches durch das Vorliegen von risikorelevanten Denk- und Verhaltensmustern verursacht wird. Sie ist angelehnt an die Global Assessment of Functioning Scale nach $\mathrm{Sa}$ und der American Psychiatric Association (1998). Die Ausprägung hat folgende Abstufungen: sehr gering, gering, moderat, stark, sehr stark. Bei einer sehr geringen Ausprägung liegen keine risikorelevanten Denk- und Verhaltensmuster vor. Bei einer geringen Ausprägung liegen risikorelevante Denk- und Verhaltensmuster vor, die hin und wieder bei spezifischen Umständen auftreten. Bei einer moderaten Ausprägung liegen risiko- 
Tab. 1 Beispielhafte Tatsituationen für die Ausprägungen

\begin{tabular}{|c|c|c|c|c|}
\hline Sehr gering & Gering & Moderat & Stark & Sehr stark \\
\hline $\begin{array}{l}\text { Ein Mann wird } \\
\text { auf der Straße } \\
\text { überfallen. Um } \\
\text { sich zu wehren, } \\
\text { schlägt er der } \\
\text { angreifenden } \\
\text { Person ins Gesicht }\end{array}$ & $\begin{array}{l}\text { Eine junge Frau ist in } \\
\text { großer Geldnot. Als ei- } \\
\text { nem Passanten } 100 € \text { aus } \\
\text { der Tasche fallen, steckt } \\
\text { sie diese ein, statt den } \\
\text { Mann darauf aufmerk- } \\
\text { sam zu machen }\end{array}$ & $\begin{array}{l}\text { In einer von Eifersucht } \\
\text { geprägten Beziehung } \\
\text { schlägt ein Mann seine } \\
\text { Freundin. Auch auf der } \\
\text { Arbeit hat er gelegentlich } \\
\text { Probleme wegen seiner } \\
\text { aufbrausenden Art }\end{array}$ & $\begin{array}{l}\text { Aufgrund seiner ausge- } \\
\text { prägten Aggressivität } \\
\text { verliert ein Mann häu- } \\
\text { fig Arbeitsstellen, gerät } \\
\text { ständig in Auseinander- } \\
\text { setzungen und schlägt } \\
\text { seine Frau und Kinder }\end{array}$ & $\begin{array}{l}\text { Aufgrund ihrer langjährigen } \\
\text { Drogenproblematik kann eine } \\
\text { Frau nicht arbeiten, hat hohe } \\
\text { Schulden und verbringt ihre Zeit } \\
\text { mit anderen Abhängigen. Um } \\
\text { ihren Konsum zu finanzieren, } \\
\text { begeht sie häufig Diebstähle }\end{array}$ \\
\hline
\end{tabular}

relevante Denk- und Verhaltensmuster vor, die bei spezifischen Umständen sehr wahrscheinlich auftreten. Bei einer starken Ausprägung liegen risikorelevante Denk- und Verhaltensmuster vor, die sich bei Umständen auswirken, die wahrscheinlich und häufig auftreten. Bei einer sehr starken Ausprägung liegen alltagsrelevante risikorelevante Denkund Verhaltensmuster vor, die unabhängig von Umständen auftreten.

In Tab. 1 sind beispielhafte Tatsituationen für die Ausprägungen ausgeführt.

\section{Passung von forensischem Bedarf und juristischen Rahmenbedingungen}

Ein wichtiger Aspekt bei der Betrachtung von deliktpräventiver Veränderung ist die Passung zwischen forensischem Bedarf und juristischen Rahmenbedingungen. Je nach Ausprägung des umwelt- und personenbezogenen Problemprofils besteht ein forensischer Bedarf. Ist dieser niedrig ausgeprägt, ist in der Regel keine forensische Intervention wie beispielsweise eine forensisch orientierte Therapie notwendig. Ist der forensische Bedarf jedoch hoch ausgeprägt, ist meist eine längerfristige intensive forensische Behandlung, mitunter auch stationär, angezeigt.

Grundsätzlich ist jedoch immer ein juristischer Rahmen im Sinne eines bestimmten Urteils oder einer Weisung gegeben, der den Umfang und die Art der Sanktion klar definiert. So kann es vorkommen, dass eine Person, bei der laut forensischem Bedarf eine Therapie deutlich angezeigt wäre, lediglich eine kurze Haftstrafe antreten muss. Ebenso kann es, wenn auch selten, vorkommen, dass eine Person mit geringem forensischem Bedarf eine stationäre Maßnahme absolvieren muss ${ }^{1}$.

Ein aus forensischer Sicht unzureichender juristischer Rahmen ist insofern problematisch, als dass angenommen werden muss, dass bis zum Ende der ausgesprochenen

\footnotetext{
${ }^{1}$ Da ein im Vergleich zum forensischen Bedarf zu großer juristischer Rahmen, wie erwähnt, sehr selten ist, wird dieser Fall im Folgenden nicht weiter miteinbezogen. Wenn also von einer unzureichenden Passung gesprochen wird, ist immer ein zu kleiner juristischer Rahmen im Vergleicht zum forensischen Bedarf gemeint.
}

Sanktion mutmaßlich keine ausreichende deliktpräventive Veränderung erfolgen kann.

\section{Ziel und Hypothesen}

Die vorab konzipierte Veränderungsmessung wurde evaluiert, um die Eignung der verwendeten Parameter für die Implementierung in ROS zu überprüfen. Hierbei wurde die Erhebung in 4 Dimensionen unterteilt: Veränderung, Rückfälligkeit, Passung und Wirksamkeit von ROS. Bezüglich der Veränderung soll betrachtet werden, inwieweit sich die betrachteten Personen am Ende der Sanktion im Vergleich zum Anfang der Sanktion entwickelt haben. Die Dimension Rückfälligkeit untersucht den Zusammenhang der Variablen am Ende der Sanktion mit der Rückfälligkeit in der Stichprobe. Im Bereich Passung wird der Zusammenhang zwischen den Variablen und der Passung forensischer Bedarf - juristischer Rahmen analysiert. In der Dimension Wirksamkeit von ROS soll in einem abschließenden Schritt ein Vergleich zu den Rückfallraten vor der Implementierung von ROS gezogen werden.

Aufgrund der großen Anzahl an Interaktionen und daraus resultierenden Hypothesen werden die aufgestellten Hypothesen in Tab. 2 dargestellt und in Kategorien eingeteilt.

\section{Methode}

\section{Datenerhebung}

Die Datenerhebung erfolgte vom 01.05.2016 bis zum 30.04.2019. Die Einschätzungen erfolgten auf Basis von Dossiers, die von den fallführenden Personen der Bewährungs- und Vollzugsdienste Zürich erstellt wurden. Die Erhebung der demografischen Daten erfolgte mithilfe von Strafregisterauszügen und Einträgen in dem Rechtsinformationssystem (RIS), einer Datenbank der Justizdirektion des Kantons Zürich.

\section{Stichprobe}

Die Stichprobe besteht aus Straftäterinnen und Straftätern des Kantons Zürich, die von den Bewährungs- und Voll- 
Tab. 2 Hypothesen

I Veränderung

Ia Die Gesamteinschätzung der personenbezogenen Bereiche der Ebenen Wollen, Wissen und Können wird im Verlauf der Sanktion positiver

Ib Die Personen befinden sich am Ende der Sanktion auf einer höheren SoC-Stufe als zu Beginn

Ic Die Gesamteinschätzung der umweltbezogenen Bereiche wird im Verlauf der Sanktion positiver

Id Die Ausprägung des personen- und umweltbezogenen Problemprofils ist am Ende der Sanktion geringer als zu Beginn

II Rückfälligkeit

IIa Personen mit einer positiveren Gesamteinschätzung der Prozessvariablen am Ende der Sanktion werden seltener rückfällig

IIb Personen auf einer höheren SoC-Stufe am Ende der Sanktion werden seltener rückfällig

IIc Personen mit einer positiveren Gesamteinschätzung der umweltbezogenen Veränderung am Ende der Sanktion werden seltener rückfällig

IId Personen mit einer geringeren Ausprägung des personen- und umweltbezogenen Problemprofils am Ende der Sanktion werden seltener rückfällig

III Passung

IIIa Personen mit hinreichender Passung haben am Ende der Sanktion eine positivere Gesamteinschätzung der Prozessvariablen als Personen mit unzureichender Passung

IIIb Personen mit hinreichender Passung befinden sich am Ende der Sanktion auf einer höheren SoC-Stufe als Personen mit unzureichender Passung

IIIc Personen mit hinreichender Passung haben am Ende der Sanktion eine positivere Gesamteinschätzung der umweltbezogenen Veränderung als Personen mit unzureichender Passung

IIId Personen mit hinreichender Passung haben am Ende der Sanktion eine niedrigere Ausprägung des personen- und umweltbezogenen Problemprofils als Personen mit unzureichender Passung

IIIe Personen mit hinreichender Passung werden seltener rückfällig als Personen mit unzureichender Passung

IV Wirksamkeit von ROS

Die Rückfälligkeit konnte mit der Einführung von ROS reduziert werden

zugsdiensten des Kanton Zürichs betreut und anschließend (bedingt) entlassen wurden. Die Entlassung führt im Rahmen des internen Prozesses dazu, dass die Fälle nach gewisser Zeit abgeschlossen werden.

Die Stichprobe umfasst Fälle, die zwischen dem 01.05.2016 und dem 30.04.2018 abgeschlossen wurden.

Aufgrund unzureichender Informationen konnte in einigen Fällen keine umfangreiche Einschätzung vorgenommen werden. Speziell konnten hier die personenbezogenen Variablen, die umweltbezogene Veränderung und die Ausprägung des Problemprofils nicht eingeschätzt werden. Die demografischen Daten, die Passung zwischen foren- sischem Bedarf und juristischen Rahmenbedingungen sowie die Rückfälligkeit wurden erhoben. Anhand von $t$-Tests wird überprüft, ob sich die Gruppe der eingeschätzten Fälle von der Gruppe der nichteingeschätzten Fälle signifikant unterscheidet.

\section{Interrater-Reliabilität}

Für die Interrater-Reliabilität-Prüfung wurden unter den Fällen mit ausreichender Aktenlage 4 Fälle zufällig ausgesucht.

Für die zufällig ausgesuchten Fälle wurden die Variablen Veränderungsmotivation, Prozessvariablen, SoC zu Beginn der Sanktion, SoC am Ende der Sanktion, umweltbezogene Veränderung, Ausprägung zu Beginn der Sanktion und Ausprägung am Ende der Sanktion eingeschätzt. Die Einschätzung wurde von 4 Angestellten der Bewährungs- und Vollzugsdienste (BVD) des Kantons Zürich durchgeführt. Bei den BVD des Kantons Zürich handelt es sich um eine einweisende Behörde, welche gemäß schweizerischer Gesetzgebung den korrekten Vollzug der gerichtlich angeordneten Sanktionen zu planen hat. In diesem Zusammenhang entscheidet sie über die Gewährung von Vollzugsprogressionen wie beispielsweise Beziehungsurlauben, Versetzungen in offenere Vollzugsregime bis hin zur bedingten Entlassung und der Anordnung von Bewährungshilfe. Um den diversen Berufshintergründen der in einer einweisenden Behörde tätigen Personen gerecht zu werden, wurden die Einschätzungen von einer Psychologin und 3 Fallverantwortlichen durchgeführt. Die Fallverantwortlichen waren hierbei Personen mit einerseits juristischem und andererseits sozialarbeiterischem Ausbildungshintergrund.

Die Übereinstimmung der Rater wird anhand von Krippendorffs $\alpha$ bestimmt. Eine perfekte Übereinstimmung würde einem Wert von $\alpha=1$ entsprechen. Krippendorf (2004) schlägt vor, dass $\alpha \geq 0,8$ sein sollte; mindestens jedoch $\geq 0,667$, um von einer gegebenen Interrater-Reliabilität ausgehen zu können.

\section{Personenbezogene Veränderungsmessung}

\section{Veränderungspotenzial}

Das Veränderungspotenzial wurde zu Beginn der Sanktion erfasst. Der erste Aspekt des Veränderungspotenzials, die Veränderungsfähigkeit, fragt nach grundlegenden, veränderungseinschränkenden Faktoren. Diese sind ausgeprägte dissoziale/impulsive Züge, Vorstrafen sowie frühere abgebrochene psychologisch/psychiatrische Behandlungen.

Der zweite Aspekt, die Veränderungsmotivation, bezieht sich auf die Ebenen Wissen, Wollen und Können in Bezug auf die deliktpräventive Bearbeitung des spezifischen Problemprofils der Person. Die Items der einzelnen Ebe- 
nen werden auf einer 4-stufigen Likert-Skala eingeschätzt. Im Anschluss an die Wertung der Items der Ebenen wird eine Gesamteinschätzung vorgenommen, ebenfalls auf einer 4-stufigen Likert-Skala. Diese bezieht neben den ItemEinschätzungen auch übergeordnet weitere Einflüsse mit ein (beispielsweise früheres Verhalten im Vollzug, mögliche Manipulationstendenzen, früheres Verhalten nach Entlassung). Die Variablen können als kaum vorhanden, etwas vorhanden, überwiegend vorhanden oder deutlich vorhanden eingeschätzt werden. Der Erhebungsbogen zu der Veränderungsmotivation ist in Abb. 1 dargestellt.

Die Abstufungen wurden wie folgt kodiert: 0: kaum vorhanden, 1: etwas vorhanden, 2: überwiegend vorhanden, 3: deutlich vorhanden. In Fällen, in denen keine Zuordnung zu 1 oder 2 erfolgen konnte, da positive und negative Faktoren sich die Waage halten, wurde eine 1,5 kodiert.

\section{Delinquenzbezogene Prozessvariablen}

Die delinquenzbezogenen Prozessvariablen beruhen auf der Veränderungsmotivation und stellen die vertiefte Erfassung im Verlauf und am Ende der Sanktion dar. Die Erhebung der Prozessvariablen gleicht der Erhebung der Veränderungsmotivation, allerdings werden die Ebenen Wissen und Können detaillierter erfasst. Der Erhebungsbogen zu den delinquenzbezogenen Prozessvariablen ist in Abb. 2 dargestellt.

Die Abstufungen wurden ebenfalls wie folgt kodiert: 0: kaum vorhanden, 1: etwas vorhanden, 2: überwiegend vorhanden, 3: deutlich vorhanden. In Fällen, in denen keine Zuordnung zu 1 oder 2 erfolgen konnte, da positive und negative Faktoren sich die Waage halten, wurde eine 1,5 kodiert.

\section{Veränderung auf Basis der Veränderungsmotivation und Prozessvariablen}

Die Veränderung wird über die Differenz zwischen Prozessvariablen und Veränderungsmotivation ermittelt. Mit einem $t$-Test für abhängige Stichproben, der die Mittelwertsunterschiede zwischen Veränderungsmotivation und Prozessvariablen betrachtet, wird die Signifikanz überprüft. Anhand von Cohens $d$ wird die Effektgröße ermittelt. Cohen (1988) schlägt für die Interpretation vor, dass $d=0,2$ einem kleinen, $d=0,5$ einem mittleren und $d=0,8$ einem großen Effekt entspricht.

\section{Stufen der Verhaltensänderung}

Die SoC wurden jeweils zu Beginn und zum Ende der Sanktion eingeschätzt. Hierbei wurden die Stufen wie folgt kodiert: 0: Absichtslosigkeit, 2: Absichtsbildung, 3: Handlung, 4: Aufrechterhaltung. In seltenen Fällen, in denen keine klare Zuordnung zu einer Stufe erfolgen konnte, wurden entsprechend Zwischenwerte gegeben $(0,5 ; 1,5 ; 2,5 ; 3,5)$.

\section{Umweltbezogene Veränderungsmessung}

Die umweltbezogene Veränderungsmessung wurde ebenfalls auf einer 4-stufigen Likert-Skala erfasst und entsprechend kodiert: 0: kaum vorhanden, 1: etwas vorhanden, 2: überwiegend vorhanden, 3: deutlich vorhanden. Die Veränderung wurde aufgrund der hohen Anzahl an unzureichenden Beschreibungen der Entwicklung folgendermaßen eingeschätzt und kodiert: 0: keine oder negative Veränderung, 0,5: leichte Verbesserung, 1: deutliche Verbesserung.

\begin{tabular}{|c|c|c|c|c|}
\hline & - & + & ++ & Keine Angabe möglich (mit Begründung) \\
\hline \multicolumn{5}{|l|}{ Wollen } \\
\hline \multicolumn{5}{|l|}{ Der Klient hat das risikorelevante Problembewusstsein zufriedenstellend erarbeitet. } \\
\hline \multicolumn{5}{|l|}{$\begin{array}{l}\text { Der Klient hat die Verantwortungsübernahme für die getroffenen risikorelevanten } \\
\text { Entscheidungen zufriedenstellend erarbeitet. }\end{array}$} \\
\hline \multicolumn{5}{|l|}{ Der Klient hat die Legalbewährungsabsicht zufriedenstellend erarbeitet. } \\
\hline \multicolumn{5}{|l|}{ Der Klient hat die risikorelevante Veränderungsbereitschaft zufriedenstellend erarbeitet. } \\
\hline \multicolumn{5}{|l|}{ Wissen } \\
\hline \multicolumn{5}{|l|}{$\begin{array}{l}\text { Der Klient hat zufriedenstellende Kenntnisse zum eigenen Problemprofil gemäß } \\
\text { Fallkonzept. }\end{array}$} \\
\hline \multicolumn{5}{|l|}{ Der Klient hat zufriedenstellende Kenntnisse zu deliktpräventiven Strategien. } \\
\hline \multicolumn{5}{|l|}{ Können } \\
\hline \multicolumn{5}{|l|}{ Der Klient kann deliktpräventive Strategien zufriedenstellend umsetzen. } \\
\hline $\begin{array}{l}\text { Gesamtbewertung: } \\
\text { Das deliktpräventive Veränderungspotenzial des Klienten ist insgesamt zufriedenstellend } \\
\text { ausgeprägt (mit Begründung). }\end{array}$ & & & & Erläuterung zur Gesamtbewertung: \\
\hline
\end{tabular}

Abb. 1 Veränderungsmotivation 


\begin{tabular}{|c|c|c|c|c|}
\hline & - & + & ++ & Keine Angabe möglich (mit Begründung) \\
\hline \multicolumn{5}{|l|}{ Wollen } \\
\hline \multicolumn{5}{|l|}{ Der Klient hat das risikorelevante Problembewusstsein zufriedenstellend erarbeitet. } \\
\hline \multicolumn{5}{|l|}{$\begin{array}{l}\text { Der Klient hat die Verantwortungsübernahme für die getroffenen risikorelevanten Entscheidungen } \\
\text { zufriedenstellend erarbeitet. }\end{array}$} \\
\hline \multicolumn{5}{|l|}{ Der Klient hat die Legalbewährungsabsicht zufriedenstellend erarbeitet. } \\
\hline \multicolumn{5}{|l|}{ Der Klient hat die risikorelevante Veränderungsbereitschaft zufriedenstellend erarbeitet. } \\
\hline \multicolumn{5}{|l|}{ Wissen } \\
\hline \multicolumn{5}{|l|}{ Der Klient hat zufriedenstellende Kenntnisse zum eigenen Problemprofil gemäß Fallkonzept. } \\
\hline \multicolumn{5}{|l|}{ Der Klient hat zufriedenstellende Kenntnisse zum eigenen Deliktverhalten gemäß Fallkonzept. } \\
\hline \multicolumn{5}{|l|}{ Der Klient hat zufriedenstellende Kenntnisse zu Frühwarnzeichen. } \\
\hline \multicolumn{5}{|l|}{ Der Klient hat zufriedenstellende Kenntnisse zu den Merkmalen der Risikosituation(en). } \\
\hline \multicolumn{5}{|l|}{ Der Klient hat zufriedenstellende Kenntnisse zu Vorbeugestrategien. } \\
\hline \multicolumn{5}{|l|}{ Der Klient hat zufriedenstellende Kenntnisse zu Bewältigungsstrategien. } \\
\hline \multicolumn{5}{|l|}{ Können } \\
\hline \multicolumn{5}{|l|}{ Der Klient hat Vorbeugestrategien zufriedenstellend umgesetzt. } \\
\hline \multicolumn{5}{|l|}{ Der Klient hat Bewältigungsstrategien zufriedenstellend umgesetzt. } \\
\hline Gesamtbewertung: & & & & Erläuterung zur Gesamtbewertung: \\
\hline $\begin{array}{l}\text { Die delinquenzbezogenen Prozessvariablen des Klienten sind insgesamt zufriedenstellend } \\
\text { ausgeprägt (mit Begründung). }\end{array}$ & & & & \\
\hline
\end{tabular}

Abb. 2 Delinquenzbezogene Prozessvariablen

\section{Ausprägung des umwelt- und personenbezogenen Problemprofils}

Die Ausprägung wurde folgendermaßen kodiert: 0: sehr stark, 1: stark, 2: moderat, 3: gering, 4: sehr gering. In Fällen, in denen keine klare Zuordnung zu den jeweiligen Ausprägungen erfolgen konnte, wurden Zwischenstufen mit $0,5,1,5,2,5$ oder 3,5 kodiert.

Die Veränderung wird über die Differenz zwischen der Ausprägung am Ende der Sanktion und der Ausprägung am Anfang der Sanktion berechnet.

\section{Passung forensischer Bedarf und juristische Rahmenbedingungen}

Die Passungen wurden folgendermaßen kodiert: 0: keine ausreichende Passung, 0,5: teilweise ausreichende Passung, 1: ausreichende Passung.

\section{Zusammenhänge zu Passung und Rückfall}

Die Zusammenhänge zwischen den personen- und umweltbezogenen Variablen und der Passung sowie dem Rückfall wurden mit einer ordinalen Regression bzw. mit einer logistischen Regression ermittelt und anhand von ,odds ratios" vorgestellt. Die Erklärungsgüte wird anhand Nagelkerke $R^{2}$ bestimmt. Hierbei entspricht $R^{2}=0,1$ einer schlechten, $R^{2}=0,3$ einer mittleren und $R^{2}=0,5$ einer guten Erklä- rungsgüte. Zur Bestimmung der Effektstärke wird Cohens $f$ herangezogen. Nach Cohen (1988) entspricht $f=0,1$ einem kleinen Effekt, $f=0,25$ einem mittleren und $f=0,4$ einem starken Effekt.

\section{Rückfallraten vor Einführung von ROS}

Die Vergleichsdaten beruhen auf Fällen, in denen zwischen 2004 und 2005 ein Geschäftsabschluss stattgefunden hat. Die im Folgenden verwendeten Daten wurden Loewe-Baur (2017) entnommen. Loewe-Baur betrachtet anhand von Strafregisterauszügen die Rückfälligkeit nach einem und nach 3 Jahren ,time at risk“.

Für den Vergleich werden die Rückfallraten für diese Erhebung anhand des Strafregisterauszugs vom 01.05.2019 herangezogen. Da die Entlassungen in den hier untersuchten Fällen größtenteils weniger als 3 Jahre zurückliegen $(\mathrm{M}=1,95$ Jahre), kann die Time at risk nicht zum 3-JahresZeitpunkt betrachtet werden. Dementsprechend wird der Vergleich in Time at risk nach einem Jahr und Time at risk nach mehr als einem Jahr unterteilt. Der Vergleich nach einem Jahr ist dementsprechend methodisch identisch mit Loewe-Baur (2017). Der Vergleich nach mehr als einem Jahr kann nur begrenzt interpretiert werden. Um die Vergleichbarkeit möglichst hoch zu halten, wird neben dem Vergleich der Rückfälle nach einem Jahr auch ein Vergleich mit dem gemittelten Rückfall nach Loewe-Baur (2017) herangezogen. Die statistische Signifikanz wird anhand von $t$ - 
Tests für unabhängige Stichproben bestimmt. Für die Effektstärke wird Cohens $d$ herangezogen.

\section{Resultate}

\section{Stichprobe und demografische Daten}

Nach Ausschluss von 2 verstorbenen Personen besteht die Gesamtstichprobe aus 302 Personen. Die Stichprobe setzt sich aus 269 Männern $(89,1 \%)$ und 33 Frauen $(10,9 \%)$ zusammen.

Das durchschnittliche Alter am Stichtag der Datenerhebung (01.05.2019) beträgt 39,29 Jahre (Standardabweichung $[\mathrm{SD}]= \pm 11,13)$. Das Alter variierte hierbei zwischen 21 Jahren und 73 Jahren.

Der Großteil der Personen besitzt eine Schweizer Staatsbürgerschaft $(n=161 ; 53,3 \%)$ oder eine andere europäische Staatsbürgerschaft $(n=76 ; 25,1 \%)$. Die verbleibenden Personen teilen sich wie folgt auf: Afrika $(n=35 ; 11,6 \%)$, Asien $(n=22 ; 7,3 \%)$, Südamerika $(n=5 ; 1,7 \%)$ und Nordamerika $(n=3 ; 1,0 \%)$.

Die deutliche Mehrheit der Personen ist ledig $(n=175$; $57,9 \%)$. Verheiratet sind 63 Personen $(20,9 \%)$ und 62 Personen $(20,5 \%)$ sind geschieden oder leben getrennt. Eine Person ist verwitwet $(0,3 \%)$, und bei einer weiteren Person konnte der Zivilstand nicht festgestellt werden.

Insgesamt 262 Personen $(86,8 \%$ ) haben eine oder mehrere Vorstrafen. Die Verteilung der Vorstrafen nach Deliktart sind in Tab. 3 abgebildet.

\section{Vergleich eingeschätzter zu nichteingeschätzter Fälle}

Aufgrund unzureichender Informationen konnte in 101 Fällen $(33,4 \%)$ keine umfangreiche Einschätzung vorgenommen werden.

Betrachtet man die Gruppenunterschiede zwischen eingeschätzten und nichteingeschätzten Fällen, so sind die Unterschiede in der Verteilung der Geschlechter $(p=0,989)$,

Tab. 3 Vorstrafen

\begin{tabular}{lll}
\hline Deliktart & $n$ & $\%$ \\
\hline Straßenverkehrsdelikte (A) & 150 & 49,7 \\
Verstöße gegen das Betäubungsmittelgesetz (A) & 158 & 52,3 \\
Eigentums- und Vermögensdelikte (A) & 185 & 61,3 \\
Verstöße gegen das Waffengesetz (A) & 61 & 20,2 \\
Verstöße gegen das Ausländergesetz (A) & 44 & 14,6 \\
Andere Delikte (A) & 81 & 26,8 \\
Gewaltdelikte & 1 & 65,2 \\
Sexualdelikte & 40 & 13,2 \\
\hline
\end{tabular}

$A$ allgemeine Delinquenz; Delikte, die keine Gewalt- oder Sexualdelikte sind des Alters $(p=0,926)$ und des Zivilstandes $(p=0,820)$ nicht signifikant. Die Verteilung der Nationalitäten weicht von der Verteilung in der Gesamtstichprobe ab. Unter den eingeschätzten Fällen befinden sich 59,7\% $(n=120)$ Schweizer und $40,3 \%(n=81)$ Nichtschweizer. Bei den nichteingeschätzten Fällen sind es 40,6\% $(n=41)$ Schweizer und $59,4 \%(n=60)$ Nichtschweizer. Der Gruppenunterschied ist signifikant $(p=0,002)$.

Bezüglich der Vorstrafen weisen $85,1 \%(n=171)$ der eingeschätzten Fälle eine Vorstrafe auf. Bei den nichteingeschätzten Fällen liegt das Vorkommen von Vorstrafen bei 90,1\% ( $n=91)$. Der Gruppenunterschied beim Vorkommen einer Vorstrafe ist nicht signifikant $(p=0,226)$. Betrachtet man die Gruppenunterschiede zwischen den einzelnen Arten von Vorstrafen, zeigt sich, dass unter den nichteingeschätzten Fällen eine Vorstrafe im Bereich Verstoß gegen das Ausländergesetz signifikant häufiger vorliegt als bei den eigeschätzten Fällen $(p<0,001)$.

Die Verteilung der Nationalitäten nach Gruppenzugehörigkeit ist in Tab. 4 abgebildet; die Verteilung der Vorstrafen nach Deliktart in Tab. 5.

Auch die Anzahl der Rückfälle laut Strafregisterauszug unterscheidet sich signifikant zwischen den Gruppen $(p<0,001)$. Bezüglich der angeklagten Rückfälle (laufende Verfahren) gibt es keinen signifikanten Gruppenunterschied $(p=0,339)$. In Tab. 6 sind die Rückfälle und angeklagten Rückfälle, aufgeteilt nach eingeschätzten und nichteingeschätzten Fällen, abgebildet.

Aufgrund der signifikanten Gruppenunterschiede müssen die eingeschätzten Fälle bei der Prüfung der Hypothesen zu Passung und Rückfälligkeit gesondert von den nichteingeschätzten Fällen betrachtet werden.

Die folgenden Ergebnisse beziehen sich dementsprechend auf die Teilstichprobe der eingeschätzten Fälle $(n=201)$.

\section{Erkenntnisse aus der Erhebung der Interrater- Reliabilität}

In der vorab durchgeführten Erhebung zur Interrater-Reliabilität wurden die vorgestellten Variablen jeweils zu Beginn und am Ende der Sanktion eingeschätzt. Es hat sich gezeigt,

Tab. 4 Nationalitäten nach Gruppenzugehörigkeit

\begin{tabular}{llllll}
\hline Nationalität & \multicolumn{2}{l}{ Eingeschätzt $(n=201)$} & & \multicolumn{2}{c}{ Nichteingeschätzt $(n=101)$} \\
\cline { 2 - 3 } & $n$ & $\%$ & $n$ & 40,6 \\
\hline Schweiz & 120 & 59,7 & 41 & 25,7 \\
Europa & 50 & 24,9 & 26 & 19,8 \\
Afrika & 15 & 7,4 & 20 & 11,9 \\
Asien & 10 & 5,0 & 12 & 1,0 \\
Nordamerika & 2 & 1,0 & 1 & 1,0 \\
Südamerika & 4 & 2,0 & 1 &
\end{tabular}


Tab. 5 Vorstrafen

\begin{tabular}{|c|c|c|c|c|}
\hline \multirow[t]{2}{*}{ Deliktart } & \multicolumn{2}{|c|}{$\begin{array}{l}\text { Eingeschätzt } \\
(n=201)\end{array}$} & \multicolumn{2}{|c|}{$\begin{array}{l}\text { Nichteingeschätzt } \\
(n=101)\end{array}$} \\
\hline & $n$ & $\%$ & $n$ & $\%$ \\
\hline $\begin{array}{l}\text { Straßenverkehrsdelikte } \\
\text { (A) }\end{array}$ & 106 & 52,7 & 44 & 43,6 \\
\hline $\begin{array}{l}\text { Verstöße gegen das } \\
\text { BetmG (A) }\end{array}$ & 108 & 53,7 & 50 & 49,5 \\
\hline $\begin{array}{l}\text { Eigentums- und } \\
\text { Vermögensdelikte } \\
\text { (A) }\end{array}$ & 122 & 60,7 & 63 & 62,4 \\
\hline $\begin{array}{l}\text { Verstöße gegen das } \\
\text { Waffengesetz (A) }\end{array}$ & 47 & 23,4 & 14 & 13,9 \\
\hline $\begin{array}{l}\text { Verstöße gegen das } \\
\text { Ausländergesetz (A) }\end{array}$ & 17 & 8,5 & 27 & 26,7 \\
\hline Andere Delikte (A) & 51 & 25,4 & 30 & 29,7 \\
\hline Gewaltdelikte & 131 & 65,2 & 66 & 65,3 \\
\hline Sexualdelikte & 28 & 13,9 & 12 & 11,9 \\
\hline
\end{tabular}

$A$ allgemeine Delinquenz; Delikte, die keine Gewalt- oder Sexualdelikte sind

dass nicht alle Variablen zu allen Zeitpunkten reliabel eingeschätzt werden konnten.

Das Veränderungspotenzial und die Prozessvariablen wurden zu Beginn und am Ende der Sanktion signifikant übereinstimmend eingeschätzt. Krippendorffs $\alpha$ beträgt hier $\alpha=0,834$ und $\alpha=0,982$.

Die SoC wurden in der Studie lediglich zum Zeitpunkt des Sanktionsbeginns signifikant übereinstimmend eingeschätzt $(\alpha=0,744)$. Zum Ende der Sanktion war die Übereinstimmung nicht signifikant $(\alpha=0,607)$. Somit ist die Voraussetzung der Reliabilität teilweise nicht erfüllt. Da für die Überprüfung einer signifikanten Veränderung im Sanktionserlauf die Reliabilität der Variablen zu beiden Zeitpunkten und für die Analyse der weiteren Hypothesen zumindest zum Ende der Sanktion gegeben sein muss, wird diese Variable nicht in die weitere Analyse miteinbezogen werden. Die Hypothesen Ib, IIb und IIIb müssen folglich als nichtbeantwortbar eingeschätzt werden.

Die Einschätzung der umweltbezogenen Veränderung wurde zu beiden Zeitpunkten nicht signifikant übereinstimmend eingeschätzt $(\alpha=0,453)$. Somit ist die Voraussetzung der Reliabilität nicht erfüllt und die umweltbezogene Veränderung kann ebenfalls nicht miteinbezogen werden. Die Hypothesen Ic, IIc und IIIc sind dementsprechend nicht beantwortbar.

Die Ausprägung des umwelt- und personenbezogenen Problemprofils wurde zum Ende der Sanktion signifikant übereinstimmend eingeschätzt $(\alpha=0,774)$. Am Anfang der Sanktion war die Übereinstimmung nicht signifikant $(\alpha=0,409)$. Diese Variable ist somit nicht in die Analyse der Veränderungsmessung miteinzubeziehen; Hypothese Id ist also nicht beantwortbar. Die reliabel eingeschätzte
Tab. 6 Rückfälle und Anklagen, aufgeteilt nach Einschätzung

\begin{tabular}{llllll}
\hline Rückfall/ & \multicolumn{2}{l}{ Eingeschätzt $(n=201)$} & & \multicolumn{2}{l}{ Nichteingeschätzt $(n=101)$} \\
\cline { 2 - 3 } Anklage & $n$ & $\%$ & $n$ & $\%$ \\
\hline Rückfall & 35 & 17,4 & 34 & 33,7 \\
Anklage $^{\mathrm{a}}$ & 19 & 9,4 & 7 & 6,9 \\
\hline
\end{tabular}

${ }^{a}$ Fälle mit Anklage, die einen auch einen verurteilten Rückfall haben, werden der Kategorie Rückfall zugeordnet

Ausprägung zum Ende der Sanktion kann jedoch in der Überprüfung der weiteren Hypothesen Verwendung finden.

\section{Veränderung}

\section{Veränderungsmotivation und Prozessvariablen}

In Tab. 7 sind die Häufigkeiten der einzelnen Ausprägungen dargestellt. Die Veränderungsmotivation stellt hierbei die Ausprägung der Veränderungsmotivation zu Beginn der Sanktion dar. Mit der Ausprägung der Prozessvariablen wird abgebildet, wie stark die risikorelevanten Denk- und Verhaltensmuster auf den Ebenen Wollen, Wissen und Können am Ende der Sanktion ausgeprägt sind.

$\mathrm{Zu}$ Beginn der Sanktion wurde die Veränderungsmotivation der deutlichen Mehrheit als kaum (54,0\%) oder etwas vorhanden $(29,7 \%)$ eingestuft. In keinem der betrachteten Fälle war die Ausprägung der Veränderungsmotivation deutlich vorhanden. Der Mittelwert der Veränderungsmotivation liegt bei $\mathrm{M}=0,59(\mathrm{SD}=0,71)$.

Am Ende der Sanktionen, wurde die Ausprägung der Prozessvariablen bei der Mehrheit der Personen $(51,8 \%$ ) als überwiegend vorhanden eingestuft. Während die Prozessvariablen ähnlich häufig kaum $(9,4 \%)$, etwas bis überwiegend $(7,1 \%)$ und deutlich vorhanden $(11,8 \%)$ waren, war auch hier die Ausprägung etwas vorhanden (20,0\%) noch verhältnismäßig häufig. Der Mittelwert der Prozessvariablen liegt bei $\mathrm{M}=1,69(\mathrm{SD}=0,79)$.

Tab. 7 Häufigkeiten der Veränderungsmotivation und der Prozessvariablen

\begin{tabular}{llllll}
\hline \multicolumn{2}{c}{ Ausprägung Veränderungsmotivation } & & \multicolumn{2}{c}{ Prozessvariablen } \\
\cline { 2 - 3 } & $n$ & $\%(n=74)$ & & $n$ & $\%(n=85)$ \\
\hline $0^{\mathrm{a}}$ & 40 & 54,0 & 8 & 9,4 \\
$1^{\mathrm{b}}$ & 22 & 29,7 & 17 & 20,0 \\
$1,5^{\mathrm{c}}$ & 4 & 5,4 & 6 & 7,1 \\
$2^{\mathrm{d}}$ & 8 & 10,8 & 44 & 51,8 \\
$3^{\mathrm{e}}$ & 0 & 0,0 & 10 & 11,8 \\
Missing & 127 & - & 116 & - \\
\hline
\end{tabular}

akaum vorhanden

betwas vorhanden

cetwas bis überwiegend vorhanden

$\mathrm{d}_{\text {überwiegend vorhanden }}$

edeutlich vorhanden 
Tab. 8 Häufigkeiten der Ausprägungen

\begin{tabular}{lll}
\hline Ausprägung & $n$ & $\%(n=189)$ \\
\hline $0^{\mathrm{a}}$ & 13 & 6,9 \\
$0,5^{\mathrm{b}}$ & 4 & 2,1 \\
$1^{\mathrm{c}}$ & 44 & 23,3 \\
$1,5^{\mathrm{d}}$ & 17 & 9,0 \\
$2^{\mathrm{e}}$ & 59 & 31,2 \\
$2,5^{\mathrm{f}}$ & 10 & 5,3 \\
$3^{\mathrm{g}}$ & 32 & 16,9 \\
$3,5^{\mathrm{h}}$ & 2 & 1,1 \\
$4^{\mathrm{i}}$ & 8 & 4,2 \\
Missing & 12 & - \\
\hline
\end{tabular}

${ }^{\mathrm{a}} \mathrm{seh}$ stark

${ }^{\mathrm{b}}$ sehr stark bis stark

${ }^{\mathrm{c}}$ stark

${ }^{d}$ stark bis moderat

emoderat

${ }^{\mathrm{f}}$ moderat bis gering

gering

${ }^{\mathrm{h}}$ gering bis sehr gering

${ }^{i}$ sehr gering

Der $t$-Test ist hochsignifikant $(p=<0,001)$. Die Effektgröße (Cohens $d$ ) liegt bei $d=-1,3$, was einem großen Effekt entspricht. Die durchschnittliche Differenz zwischen den Prozessvariablen und der Veränderungsmotivation liegt bei $\mathrm{M}=0,92(\mathrm{SD}=0,82)$. Im Schnitt verbessert sich die Ausprägung der Variablen im Verlauf der Sanktion also um eine Stufe.

\section{Ausprägung des personen- und umweltbezogenen Problemprofils}

In Tab. 8 sind die Häufigkeiten der einzelnen Ausprägungen am Ende der Sanktion dargestellt. Aufgrund der fehlenden Interrater-Reliabilität werden die Ausprägungen zu Beginn der Sanktion nicht berücksichtigt.

Am Ende der Sanktion war das Problemprofil in den meisten Fällen moderat $(31,2 \%)$ ausgeprägt. Häufig waren außerdem die Ausprägungen stark (23,3\%) oder gering $(16,9 \%)$. Weniger häufig ausgeprägt waren die Zwischenstufen (sehr stark bis stark $=2,1 \%$, stark bis moderat $=9,0 \%$, moderat bis gering $=5,3 \%$ und gering bis sehr gering $=1,1 \%)$. Der Mittelwert der Ausprägung am Ende der Sanktion liegt bei $\mathrm{M}=1,85(\mathrm{SD}=0,97)$.

\section{Rückfälligkeit}

\section{Signifikanztests}

Die Signifikanztests wurden mit den laut Strafregister verurteilten Rückfällen durchgeführt. Anklagen wurden nicht miteinbezogen. In Tab. 9 sind die Zusammenhänge der Variablen mit Rückfall dargestellt.
Tab. 9 Zusammenhänge, Rückfall

\begin{tabular}{|c|c|c|c|c|}
\hline Zusammenhang & $p$ & $\begin{array}{l}\text { Odds } \\
\text { ratio }\end{array}$ & Nagelkerke $R^{2}$ & Cohen's $f$ \\
\hline Rückfall × Prozess & $0,007 * *$ & 0,41 & 0,12 & 0,02 \\
\hline $\begin{array}{l}\text { Rückfall × Ausprä- } \\
\text { gung }\end{array}$ & $<0,001 * * *$ & 2,79 & 0,17 & 0,03 \\
\hline
\end{tabular}

Personen mit positiverer Ausprägung der Prozessvariablen am Ende der Sanktion haben eine um 59\% gesenkte Chance $(\mathrm{OR}=0,41)$, rückfällig $\mathrm{zu}$ werden. Die Erklärungsgüte des Zusammenhangs ist hierbei moderat $\left(R^{2}=0,12\right)$ bei einer geringen Effektstärke von $f=0,02$.

Bezüglich der Ausprägung des personen- und umweltbezogenen Problemprofils, ist die Chance, rückfällig zu werden, bei den Personen mit einer höheren Ausprägung am Ende der Sanktion um 2,79 erhöht. Die Erklärungsgüte des Zusammenhangs ist moderat $\left(R^{2}=0,17\right)$ bei einer geringen Effektstärke von $f=0,03$.

\section{Passung}

In Tab. 10 sind die Einschätzungen der Passung zwischen forensischem Bedarf und juristischen Möglichkeiten abgebildet. Hier werden zunächst auch die Fälle abgebildet, in denen keine Einschätzung der weiteren Variablen vorgenommen werden konnte. Anschließend wird jedoch erneut auf die Teilstichprobe der eingeschätzten Fälle Bezug genommen.

Unter den nichteingeschätzten Fällen war die Passung zwischen forensischem Bedarf und juristischen Rahmenbedingungen etwas häufiger ausreichend (56,1\%) als nichtausreichend $(42,7 \%)$. Bei den Fällen, in denen eine Einschätzung möglich war, war eine ausreichende Passung $(70,1 \%)$ deutlich häufiger als eine nichtausreichende Passung $(28,3 \%)$.

Die Zusammenhänge zwischen Passung und Prozessvariablen sowie zwischen Passung und Rückfall sind nicht signifikant. Der Zusammenhang zwischen Passung und Ausprägung des Problemprofils am Ende der Sanktion ist zwar

Tab. 10 Häufigkeiten der Passungen

\begin{tabular}{llllll}
\hline Passung & \multicolumn{2}{l}{ Nichteingeschätzte Fälle } & & \multicolumn{2}{l}{ Eingeschätzte Fälle } \\
\cline { 2 - 3 } \cline { 5 - 5 } & $n$ & & $n(n=82)$ & & $\begin{array}{l}\% \\
(n=187)\end{array}$ \\
\hline $0^{\mathrm{a}}$ & 35 & 42,7 & 53 & 28,3 \\
$0,5^{\mathrm{b}}$ & 1 & 1,2 & 3 & 1,6 \\
$1^{\mathrm{c}}$ & 46 & 56,1 & 131 & 70,1 \\
Missing & 27 & - & 14 & - \\
\hline
\end{tabular}

${ }^{a}$ keine ausreichende Passung

$\mathrm{b}_{\text {teilweise ausreichende Passung }}$

${ }^{c}$ ausreichende Passung 
hochsignifikant $(p<0,001)$, weist jedoch eine geringe Erklärungsgüte $\left(R^{2}=0,102\right)$ auf.

\section{Wirksamkeit von ROS}

In Tab. 11 und 12 sind die Rückfallraten nach Loewe-Baur (2017), also die Rückfallraten vor der Einführung von ROS aus den Jahren 2004 und 2005, nach einem Jahr und gemittelter TaR (2 Jahre) mit den Rückfallraten aus dieser Erhebung nach einem Jahr TaR und mehr als einem Jahr TaR gegenübergestellt. Hierbei werden die eingeschätzten Fälle, die nichteingeschätzten Fälle sowie die kombinierte Stichprobe aus eingeschätzten und nichteingeschätzten Fällen berücksichtigt. In Tab. 13 sind die statistischen Kennwerte der $t$-Tests aufgeführt.

\section{Rückfälligkeit in der kombinierten Stichprobe}

Vergleicht man die Rückfallraten mit denen von LoeweBaur (2017) nach einem Jahr Time at risk, so kann man sehen, dass die Rückfallraten in der kombinierten Stichprobe aus eingeschätzten und nichteingeschätzten Fällen von 34,7 auf $18,2 \%$ gesunken sind. Der Gruppenunterschied ist hochsignifikant $(p<0,001)$, bei einer kleinen Effektstärke von $d=0,4$. Vergleicht man die gemittelten Rückfallraten von Loewe-Baur (2017) mit den Rückfallraten nach Einführung von ROS, ist ein noch gravierenderer Unterschied zu erkennen. So ist der Rückfall in der kombinierten Stichprobe um etwa die Hälfte geringer (von 44,3 auf $22,9 \%, p<0,001)$. Dies entspricht einem mittleren Effekt von $d=0,5$.
Insbesondere der Rückfall im Bereich der Allgemeindelinquenz ist gesunken (von 30,4 bzw. 37,3 auf 14,6 bzw. $18,9 \%)$, bei einem jeweils signifikanten Gruppenunterschied $(p<0,001)$ und kleiner Effektstärke $(d=0,4)$. Die Rückfallraten im Bereich der Gewalt- und Sexualdelinquenz nach einem Jahr sind annährend gleich geblieben (von 4,3 auf 3,6\%), bei nichtsignifikantem Gruppenunterschied $(p=0,649)$. Beim Vergleich mit der gemittelten TaR sind die Rückfallraten gesunken (von 6,8 auf 4,0\%). Allerdings ist diese Senkung nicht signifikant $(p=0,061)$. Ergänzend ist zu erwähnen, dass es in der gesamten Stichprobe nach der Einführung von ROS keinen Rückfall im Bereich der Sexualdelinquenz gab.

\section{Rückfälligkeit bei nichteingeschätzten Fällen}

Bei den nichteingeschätzten Fällen ist die Rückfälligkeit im Vergleich zu einem Jahr TaR bei Loewe-Baur (2017) zwar annährend gleichgeblieben (von 34,7 auf 28,7\%,p=0,228), jedoch im Vergleich zur gemittelten Rückfälligkeit deutlich geringer (von 44,3 auf 33,7\%, $p=0,043, d=0,2$ ). Im Bereich der Allgemeindelinquenz stellt sich ein ähnliches Bild dar (von 30,4 auf $21,8 \%$ bei $p=0,059$ bzw. von 37,3 auf $25,7 \%$ bei $p=0,016, d=0,2$ ). Bei den Gewalt- und Sexualdelikten hingegen sind die Gruppenunterschiede jeweils nicht signifikant $(4,3 \%$ zu 7,8\%, $p=0,320$ nach einem Jahr, bzw. 6,8\% zu 7,9\%, $p=0,683$ gemittelt).

Tab. 11 Vergleich der Rückfallraten nach TaR, 1 Jahr

\begin{tabular}{|c|c|c|c|c|}
\hline Rückfälligkeit & $\begin{array}{l}\text { Loewe-Baur (1 Jahr) } \\
n=632\end{array}$ & $\begin{array}{l}\text { Eingeschätzt } \\
n=201\end{array}$ & $\begin{array}{l}\text { Nichteingeschätzt } \\
n=101\end{array}$ & $\begin{array}{l}\text { Kombinierte Stichprobe } \\
n=302\end{array}$ \\
\hline$\overline{\text { Kein Rückfall }}$ & $413(65,3 \%)$ & $175(87,1 \%)$ & $72(71,3 \%)$ & $247(81,8 \%)$ \\
\hline Rückfall & $219(34,7 \%)$ & $26(12,9 \%)$ & $29(28,7 \%)$ & $55(18,2 \%)$ \\
\hline $\mathrm{G} / \mathrm{S}^{\mathrm{a}}$ & $27(4,3 \%)$ & $4(2,0 \%)$ & $7(6,9 \%)$ & $11(3,6 \%)$ \\
\hline Allgemein ${ }^{\mathrm{b}, \mathrm{c}}$ & $192(30,4 \%)$ & $22(10,9 \%)$ & $22(21,8 \%)$ & $44(14,6 \%)$ \\
\hline
\end{tabular}

${ }^{\mathrm{a}}$ Gewalt- oder Sexualdelikt

${ }^{b}$ Delikte, die keine Gewalt- oder Sexualdelikte sind

${ }^{\mathrm{c}}$ Wurde sowohl ein Gewalt- oder Sexualdelikt als auch ein allgemeines Delikt begangen, wurde die Person der Kategorie G/S zugeordnet

Tab. 12 Vergleich der Rückfallraten nach TaR, mehr als 1 Jahr

\begin{tabular}{|c|c|c|c|c|}
\hline Rückfälligkeit & $\begin{array}{l}\text { Loewe-Baur (gemittelt) } \\
n=632\end{array}$ & $\begin{array}{l}\text { Eingeschätzt } \\
n=201\end{array}$ & $\begin{array}{l}\text { Nichteingeschätzt } \\
n=101\end{array}$ & $\begin{array}{l}\text { Kombinierte Stichprobe } \\
n=302\end{array}$ \\
\hline Kein Rückfall & $352(55,7 \%)$ & $166(82,6 \%)$ & $67(66,3 \%)$ & $233(77,1 \%)$ \\
\hline Rückfall & $279(44,3 \%)$ & $35(17,4 \%)$ & $34(33,7 \%)$ & $69(22,9 \%)$ \\
\hline $\mathrm{G} / \mathrm{S}^{\mathrm{a}}$ & $43(6,8 \%)$ & $4(2,0 \%)$ & $8(7,9 \%)$ & $12(4,0 \%)$ \\
\hline Allgemein ${ }^{\mathrm{b}, \mathrm{c}}$ & $236(37,3 \%)$ & $31(15,4 \%)$ & $26(25,7 \%)$ & $57(18,9 \%)$ \\
\hline
\end{tabular}

${ }^{\mathrm{a}}$ Gewalt- oder Sexualdelikt

bDelikte, die keine Gewalt- oder Sexualdelikte sind

${ }^{c}$ Wurde sowohl ein Gewalt- oder Sexualdelikt als auch ein allgemeines Delikt begangen, wurde die Person der Kategorie G/S zugeordnet 
Tab. 13 Statistische Kennwerte

\begin{tabular}{llllll}
\hline $\begin{array}{l}\text { Verglichene } \\
\text { Stichproben }\end{array}$ & Aspekt & $t$ & $D f$ & $p$ & $d$ \\
\hline LB $^{\mathrm{a}}-$ Kombi & & & & & \\
(1 Jahr) & Rückfall & 5,627 & 716 & $<0,001^{* * *}$ & 0,4 \\
& G/S & 0,455 & 932 & 0,649 & - \\
& Allgemein & 5,778 & 751 & $<0,001^{* * * *}$ & 0,4 \\
LB - E & Rückfall & 7,152 & 475 & $<0,001^{* * *}$ & 0,5 \\
(1 Jahr) & G/S & 1,791 & 486 & 0,074 & - \\
& Allgemein & 6,776 & 495 & $<0,001^{* * *}$ & 0,5 \\
LB - NE & Rückfall & 1,211 & 137 & 0,228 & - \\
(1 Jahr) & G/S & $-0,998$ & 121 & 0,320 & - \\
& Allgemein & 1,904 & 142 & 0,059 & - \\
LB - Kombi & Rückfall & 6,816 & 690 & $<0,001^{* * *}$ & 0,5 \\
(Mittel) & G/S & 1,877 & 744 & 0,061 & - \\
& Allgemein & 6,227 & 717 & $<0,001^{* * *}$ & 0,4 \\
LB - E & Rückfall & 8,025 & 436 & $<0,001^{* * *}$ & 0,6 \\
(Mittel) & G/S & 3,421 & 617 & $0,001^{* * *}$ & 0,2 \\
& Allgemein & 6,853 & 446 & $<0,001^{* * * *}$ & 0,5 \\
LB - NE & Rückfall & 2,046 & 137 & $0,043^{*}$ & 0,2 \\
(Mittel) & G/S & $-0,409$ & 731 & 0,683 & - \\
& Allgemein & 2,428 & 142 & $0,016^{*}$ & 0,2 \\
\hline
\end{tabular}

$* p<0.05 \% ; * * p<0.01 \% ; * * * p<0.001 \%$

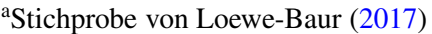

${ }^{\mathrm{b}}$ Kombinierte Stichprobe

${ }^{c}$ Eingeschätzte Fälle

${ }^{\mathrm{d}}$ Nichteingeschätzte Fälle

\section{Rückfälligkeit bei eingeschätzten Fällen}

Bei den eingeschätzten Fällen sind die Rückfallraten in allen Bereichen und $\mathrm{zu}$ beiden Zeitpunkten deutlich geringer seit Einführung von ROS. So ist die generelle Rückfälligkeit um die Hälfte bzw. um das Dreifache gesunken (von 34,7 bzw. 44,3 auf 12,9 bzw. 17,4\%). Ebenso ist die Rückfälligkeit bei Allgemeindelinquenz um die Hälfte bzw. um das Dreifache gesunken (von 30,4 bzw. 21,8 auf 10,9 bzw. $15,4 \%$ ). Alle Gruppenunterschiede sind jeweils hochsignifikant $(p<0,001)$, bei mittlerer Effektstärke $(d=0,5$ bzw. 0,6 für Rückfall nach gemittelter TaR). Die Rückfälligkeit im G- und S-Bereich ist bei den eingeschätzten Fällen im Vergleich zur TaR von einem Jahr bei Loewe-Baur (2017) nicht signifikant gesunken (von 4,3 auf $2 \%, p=0,074$ ). Im Vergleich zur gemittelten TaR ist der Gruppenunterschied jedoch hochsignifikant (von 6,8 auf $2 \%, p=0,001$ ), bei kleiner Effektstärke $(d=0,2)$.

\section{Diskussion}

Für eine verbesserte Übersicht sind die Hypothesen nachfolgend in Kurzform und nach Dimension in Tab. 14 dargestellt. Zusätzlich ist die Erkenntnis vermerkt, ob die Hypothese bestätigt oder nicht bestätigt werden kann. Als nicht-
Tab. 14 Hypothesen mit Kurzbezeichnung und Erkenntnis

\begin{tabular}{|c|c|c|}
\hline \multicolumn{3}{|c|}{ I Veränderung } \\
\hline Ia & $\begin{array}{l}\text { Positivere Ausprägung Prozessvariablen } \\
\text { am Ende }\end{array}$ & Bestätigt \\
\hline $\mathrm{Ib}$ & Höhere SoC-Stufe am Ende & $\begin{array}{l}\text { Nicht beant- } \\
\text { wortbar }\end{array}$ \\
\hline Ic & $\begin{array}{l}\text { Umweltbezogene Bereiche am Ende } \\
\text { positiver }\end{array}$ & $\begin{array}{l}\text { Nicht beant- } \\
\text { wortbar }\end{array}$ \\
\hline Id & $\begin{array}{l}\text { Ausprägung des Problemprofils am Ende } \\
\text { geringer }\end{array}$ & $\begin{array}{l}\text { Nicht beant- } \\
\text { wortbar }\end{array}$ \\
\hline \multicolumn{3}{|c|}{ II Rückfälligkeit } \\
\hline IIa & $\begin{array}{l}\text { Positivere Ausprägung Prozessvariablen: } \\
\text { seltener rückfällig }\end{array}$ & Bestätigt \\
\hline $\mathrm{IIb}$ & $\begin{array}{l}\text { Höhere SoC-Stufe am Ende: seltener } \\
\text { rückfällig }\end{array}$ & $\begin{array}{l}\text { Nicht beant- } \\
\text { wortbar }\end{array}$ \\
\hline IIc & $\begin{array}{l}\text { Positivere Gesamteinschätzung Umwelt } \\
\text { am Ende: seltener rückfällig }\end{array}$ & $\begin{array}{l}\text { Nicht beant- } \\
\text { wortbar }\end{array}$ \\
\hline IId & $\begin{array}{l}\text { Geringere Ausprägung Problemprofil am } \\
\text { Ende: seltener rückfällig }\end{array}$ & Bestätigt \\
\hline \multicolumn{3}{|c|}{ III Passung } \\
\hline IIIa & $\begin{array}{l}\text { Hinreichende Passung: positivere Aus- } \\
\text { prägung, Prozessvariablen }\end{array}$ & $\begin{array}{l}\text { Nicht bestä- } \\
\text { tigt }\end{array}$ \\
\hline IIIb & $\begin{array}{l}\text { Hinreichende Passung: höhere SoC-Stufe } \\
\text { am Ende }\end{array}$ & $\begin{array}{l}\text { Nicht beant- } \\
\text { wortbar }\end{array}$ \\
\hline IIIc & $\begin{array}{l}\text { Hinreichende Passung: positivere Ge- } \\
\text { samteinschätzung, Umwelt am Ende }\end{array}$ & $\begin{array}{l}\text { Nicht beant- } \\
\text { wortbar }\end{array}$ \\
\hline IIId & $\begin{array}{l}\text { Hinreichende Passung: geringere Aus- } \\
\text { prägung, Problemprofil am Ende }\end{array}$ & Bestätigt \\
\hline IIIe & $\begin{array}{l}\text { Hinreichende Passung: weniger Rückfäl- } \\
\text { ligkeit }\end{array}$ & $\begin{array}{l}\text { Nicht bestä- } \\
\text { tigt }\end{array}$ \\
\hline \multicolumn{3}{|c|}{ IV Wirksamkeit von ROS } \\
\hline- & $\begin{array}{l}\text { Rückfälligkeit mit Einführung von ROS } \\
\text { reduziert }\end{array}$ & Bestätigt \\
\hline
\end{tabular}

beantwortbar sind die Hypothesen gekennzeichnet, welche nicht überprüft wurden, weil die Variablen nicht reliabel eingeschätzt werden konnten.

\section{Veränderung}

Hypothese Ia (Veränderung, Prozessvariablen) kann bestätigt werden. Dementsprechend wird die Gesamteinschätzung der personenbezogenen Bereiche der Ebenen Wollen, Wissen und Können im Verlauf der Sanktion im Schnitt positiver. Konkret wird die Ausprägung um eine Einheit verbessert. Dies kann als Hinweis darauf gewertet werden, dass während der Sanktion zielführend mit den Straftäterinnen und Straftätern gearbeitet wird.

Wie bereits im ROS verankert, beinhaltet dieser Ansatz nicht nur therapeutische und psychologische Arbeit (personenbezogene Aspekte), sondern auch sozialarbeiterische und umweltbezogene Aspekte, die gezielt an der Lebenssituation der Straftäterinnen und Straftätern ansetzen. Die Veränderung dieser umweltbezogenen Aspekte kann entsprechend den Erkenntnissen aus der IRR-Erhebung jedoch 
mit der hier verwendeten Erfassungsmethode nicht reliabel eingeschätzt werden. Mögliche Gründe hierfür sind einerseits die große Diversität der Aspekte und andererseits der sich verändernde Fokus während der Sanktion. Beide Gründe können mitunter zu einer schwer quantifizierbaren Aktenlage führen. Umweltbezogene Aspekte sind potenziell schnell veränderbar. So kann beispielsweise eine zu Beginn stabile Familienkonstellation während des Vollzugs belastet werden. Dementsprechend wäre dieser Aspekt einer deutlichen Schwankung ausgesetzt und folglich schwer reliabel zu quantifizieren.

Auch Veränderungen in der finanziellen und der Arbeitssituation sind nicht einfach einzuschätzen, weil sie häufig zu Beginn des Vollzugs eine untergeordnete Rolle spielen und demnach wenig dazu dokumentiert wird. Gegen Ende des Vollzugs rücken Umweltfaktoren jedoch generell stärker in den Fokus, um eine potenzielle Bewährungshilfe sinnvoll und unterstützend gestalten zu können. Der verschobene Fokus kann dazu führen, dass die Aktenlage keine oder nur eine eingeschränkte Veränderungseinschätzung zulässt. Entsprechend umgekehrt stellt es sich bei den SoC dar, die einen starken Fokus auf Veränderungsbereitschaft legen. Diese wird insbesondere zu Beginn der Sanktion intensiv betrachtet; zum Ende der Sanktion spielt sie dann eine eher untergeordnete Rolle und wird folglich weniger ausführlich dokumentiert.

\section{Rückfälligkeit}

Hypothese IIa und IId (Rückfälligkeit, Prozessvariablen und Ausprägung) kann ebenfalls bestätigt werden. Sowohl eine höhere Ausprägung der Prozessvariablen als auch eine geringere Ausprägung des personen- und umweltbezogenen Problemprofils hängt signifikant mit geringerer Rückfälligkeit zusammen.

Die Bestätigung der Hypothesen liefert einen Hinweis darauf, dass die Integration der Variablen in das Konzept des ROS sinnvoll sein könnte. Prozessvariablen wie auch die Ausprägung des personen- und umweltbezogenen Problemprofils scheinen in Bezug auf die Bewertung potenzieller Rückfälligkeit, vielversprechende prädiktive Variablen zu sein, auch wenn die gefundenen Effektstärken eher gering sind.

\section{Passung}

Hypothese III (Passung) kann nur teilweise bestätigt werden. Die Hypothesen IIIa und IIIc werden verworfen. Folglich muss angenommen werden, dass kein Zusammenhang zwischen der Passung von forensischem Bedarf und juristischen Rahmenbedingungen und der Ausprägung der Prozessvariablen besteht; ebenso wie kein Zusammenhang zwischen der Passung und Rückfälligkeit besteht.
Dahingegen scheint es einen Zusammenhang zwischen der Passung und der Ausprägung des personen- und umweltbezogenen Problemprofils zu geben. Demnach kann angenommen werden, dass sich eine ausreichende Passung zwischen forensischem Bedarf und juristischen Rahmenbedingungen positiv auf die Veränderung der verschiedenen personen- und umweltbezogenen Variablen auswirkt. Aufgrund der geringen Erklärungsgüte müssen diese Ergebnisse jedoch zurückhaltend interpretiert werden.

Relativierend ist im ROS verankert, dass auch bei unzureichender Passung darauf geachtet wird, die vollzugspraktischen Möglichkeiten möglichst gut zu nutzen, um dennoch am individuellen Problemprofil und dem risikorelevanten Veränderungsbedarf zu arbeiten.

Folgerichtig sollte also weiterhin eine möglichst adäquate Passung angestrebt werden und, bei unzureichender Passung, unter Nutzung sämtlicher vollzugspraktischer Möglichkeiten, die delinquenzbezogenen personen- und umweltbezogenen Bereiche bearbeitet werden.

\section{Wirksamkeit von ROS}

Hypothese IV (Wirksamkeit von ROS) kann bestätigt werden. Vergleicht man die Rückfallraten nach Loewe-Baur (2017) mit den aktuellen Rückfallraten, so kann man feststellen, dass die Rückfälligkeit in der kombinierten Stichprobe aus eingeschätzten und nichteingeschätzten Fällen von $34,7 \%(\mathrm{TaR}=1 \mathrm{Jahr}$ ) bzw. 44,3\% (gemittelte TaR) vor der Einführung von ROS auf insgesamt 22,9\% nach der Einführung von ROS gesunken ist. Betrachtet man spezifisch die eingeschätzten Fälle, sinkt die Rückfälligkeit sogar auf $17,4 \%$. Auch bei der Rückfälligkeit der nichteingeschätzten Fälle ist eine Senkung von $10 \%$ zu beobachten (von gemittelten 44,3\% bei Loewe-Baur (2017) zu 33,7\%). Allerdings ist diese Senkung erst signifikant, wenn man die Rückfallraten vor Einführung von ROS mit der gemittelten TaR vergleicht. Mögliche Gründe hierfür werden im Folgenden näher erläutert.

Betrachtet man die Vorgeschichte der nichteingeschätzten Fälle, kann man sehen, dass hier anteilig gleich viele Personen wegen eines Gewaltdelikts verurteilt wurden wie bei den eingeschätzten Fällen $(65,2 \%$ der eingeschätzten zu $65,3 \%$ der nichteingeschätzten Fälle). Es kann vermutet werden, dass die nichteingeschätzten Personen mit Gewaltdelikt in der Vorgeschichte häufiger einschlägig rückfällig geworden sind. Dafür spräche der erhöhte Anteil der Rückfälle im Bereich Gewaltdelikte der nichteingeschätzten Fälle $(7,8 \%$ der nichteingeschätzten zu 2,0\% der eingeschätzten Fälle). Bezüglich des verhältnismäßig geringen Unterschieds in den Rückfallzahlen im Bereich der Gewaltdelikte gilt es Folgendes zu berücksichtigen.

Einerseits haben Gewalt- und Sexualdelikte (G/S-Delikte) eine geringere Basisrate als Allgemeindelinquenz - 
sprich, sie werden seltener begangen. Bereits eine geringfügige Senkung der Zahlen ist also als Erfolg zu sehen.

Andererseits ist es so, dass die Fälle häufig dann nicht eingeschätzt werden konnten, wenn keine bedingte Entlassung gewährt wurde. Gemäß schweizerischer Gesetzgebung ist dadurch die juristische Voraussetzung für die Anordnung von Bewährungshilfe nicht gegeben. Dies verändert die Aktenlage insofern, als dass den Abschlussberichten der Bewährungshilfe in der Regel die für eine Einschätzung notwendigen Informationen entnommen werden können. Die Abschlussberichte aus den Institutionen dahingegen haben einen anderen Fokus und sind nicht so umfangreich, weswegen sie meist keine ausreichende Informationsgrundlage für eine Einschätzung der Variablen darstellen. Eine bedingte Entlassung wird häufig dann verwehrt, wenn das Verhalten während des Vollzugs als mangelhaft eingeschätzt wird; wenn beispielsweise der Umgang mit dem Personal der Vollzugseinrichtung oder Mitinsassen unangemessen war oder bereits neue Delikte begangen wurden. Dies ist häufig ein Zeichen für eine problematische Einstellung in Bezug auf Normen und Gesetze und dementsprechend ein Indikator für künftige Delinquenz.

Zusätzlich kann Bewährungshilfe auch dann nicht angeordnet werden, wenn kein Resozialisierungsauftrag in die Schweiz besteht. Dies ist dann der Fall, wenn eine gerichtlich angeordnete Landesverweisung oder eine migrationsrechtliche Ausweisung im Raum steht. Nicht selten ist es jedoch so, dass diese Personen aus politischen oder völkerrechtlichen Gründen nicht ausgeschafft werden können (beispielsweise aufgrund von Ausschaffungen in Kriegsund Krisengebiete oder wenn das Heimatland die Aufnahme verweigert). Unter den nichteingeschätzten Fällen befinden sich einerseits überproportional viele Personen ohne Schweizer Staatsbürgerschaft und andererseits viele Personen ohne angeordnete Bewährungshilfe. Ebenfalls ist in dieser Gruppe das Vorkommen von Verstößen gegen das Ausländergesetz (das das Aufenthaltsbestimmungsrecht in der Schweiz regelt) sowohl in der Vorstrafe als auch in der Rückfälligkeit deutlich höher. Insbesondere bezüglich der Rückfälligkeit können diese Verstöße nur von Personen begangen worden sein, die sich illegal in der Schweiz aufgehalten haben oder illegal in die Schweiz zurückgekehrt sind. Die Definition der exakten Zahl der Personen, die irgendwann im Verlauf des Untersuchungszeitraumes tatsächlich des Landes verwiesen wurden, ist aufgrund der migrationsrechtlichen Praxis in der Schweiz erheblich erschwert (Einspruchsmöglichkeiten mit aufschiebender Wirkung der Ausschaffung). Es bleibt grundsätzlich abzuwägen, ob Personen, die des Landes verwiesen werden sollen, in die Analyse einbezogen oder ausgeschlossen werden. In der vorliegenden Stichprobe hat der Miteinbezug oder Ausschluss dieser Population, aufgrund der oben dargelegten Zusammenhänge, kaum Einfluss auf die Rückfalldaten der einge- schätzten Personen. In Bezug auf die nichteingeschätzten Personen sind folgende Auswirkungen anzunehmen. Bei Nichtberücksichtigung werden potenziell Personen ausgeschlossen, die in der Schweiz rückfällig werden, weil sie das Land nicht verlassen (können). Zudem können Rückfälle, die im Ausland begangen wurden, nicht erfasst werden. Bei einem Miteinbezug bleiben diese Rückfälle im Ausland ebenfalls unerkannt. Erneute Delinquenz von Personen, die trotz geplanter Ausweisung in der Schweiz bleiben, fließt jedoch in die Berechnungen mit ein. In beiden Fällen wird die tatsächliche Rückfälligkeit potenziell unterschätzt. In Loewe-Baur (2017) wurden Personen, welche die Schweiz nach Verbüßen der Sanktion potenziell verlassen müssen, aus den Berechnungen zur Rückfälligkeit ausgeschlossen, in der vorliegenden Erhebung hingegen mitberücksichtigt, weil obige Erläuterungen nahelegen, dass die Verzerrung dadurch geringer ausfällt.

Grundsätzlich lässt sich festhalten, dass der Unterschied in den Rückfallraten zwischen eingeschätzten und nichteingeschätzten Fällen ein potenzieller Hinweis darauf ist, dass, insbesondere im Bereich der Allgemeindelinquenz, eine Bewährungshilfe einen zusätzlichen, wertvollen Beitrag zur Senkung des Rückfallrisikos leistet.

\section{Kritik}

Bezüglich der Validität lässt sich festhalten, dass die Variablen zwar alle auf ausgiebiger Literaturbasis beruhen (hierzu Treuthardt und Kröger 2021) und an andere bestehende, validierte Tests angelehnt sind, jedoch keine explizite Validierung derselben stattgefunden hat.

Die Untersuchung der Interrater-Reliabilität kann nur eingeschränkt interpretiert werden. Dies ist der Tatsache geschuldet, dass die Einschätzung durch die Rater lediglich in 4 der ursprünglich 201 eingeschätzten Fällen erfolgen konnte. Wünschenswert wäre die Einschätzung einer größeren Stichprobe. Jedoch ist es üblich und legitim, bei zeit- oder kostenaufwendigen Untersuchungen, die Interrater-Reliabilität anhand kleinerer Stichproben zu betrachten (Hallgren 2012). Aufgrund der hohen Zeitintensität der Einschätzung der Variablen wurde dies hier so praktiziert.

Bezüglich der Interrater-Reliabilität zeigt sich generell, dass nicht alle ursprünglich zur Veränderungsmessung konstruierten Variablen zu beiden Messzeitpunkten übereinstimmend eingeschätzt werden konnten. Ein entscheidender Grund für die Unterschiede der Reliabilität zu den verschiedenen Zeitpunkten könnte, wie oben ausgeführt, sein, dass die Bewertung auf der Basis sehr unterschiedlicher Informationsgrundlagen erfolgten musste. Dies ist in den unterschiedlichen Foci zu Beginn bzw. am Ende einer Sanktion begründet.

Ergänzend ist zu erwähnen, dass ein reger Austausch zwischen den fallbeteiligten Personen im ROS-Prozess ver- 
ankert ist. Dies bedeutet insbesondere, dass Fälle regelmäBig besprochen und Einschätzungen begründet werden müssen. Wichtig ist hierbei, dass alle Beteiligten dieselben Begrifflichkeiten und Konzepte verwenden. Explizit bedeutet das nicht, dass jede Person dieselbe Einschätzung treffen soll. Eine Diskrepanz zwischen Wertungen bedeutet also weniger ein Problem, sondern kann vielmehr als Hinweis für einen intensiveren Austausch gewertet werden.

Ein weiterer Kritikpunkt ist, dass zwar die Zusammenhänge der einzelnen Variablen im Verlauf sowie zur Passung und zum Rückfall untersucht werden konnten, die Erstellung eines Modells zur Varianzaufklärung jedoch nicht möglich war. Dies ist darin begründet, dass die Variablen untereinander hoch korrelieren und dementsprechend nicht unabhängig voneinander in einem Modell gerechnet werden können.

\section{Fazit und Ausblick}

Die konzipierte Veränderungsmessung scheint grundsätzlich sinnvoll zu sein, erkennbar an der Bestätigung des Großteils der auswertbaren Hypothesen. Sowohl die personenbezogenen Veränderungsmaße des Veränderungspotenzials bzw. der Prozessvariablen als auch die übergeordnete Ausprägung des personen- und umweltbezogenen Problemprofils weisen stabile, wenn auch geringe, Zusammenhänge zur Rückfälligkeit auf.

Bei einer potenziellen Einführung der vorgestellten Variablen in ROS wäre einerseits entscheidend, eine einheitlichere Bewertungsgrundlage zu schaffen. Dies könnte beispielsweise eine weitere Standardisierung von Eintrittserhebungen, Fallbesprechungen und Berichterstattungen umfassen. Andererseits sollte die Einschätzung der Variablen gezielt geschult werden. Schulungen sind bereits ein wichtiger Bestandteil des ROS-Konzepts und werden regelmäßig für die (neuen) Mitarbeitenden durchgeführt, um den Qualitätsstandards gerecht zu werden. Eine Erweiterung könnte nun einerseits eine einheitlichere Datengrundlage in den Dossiers bewirken. Andererseits könnte so - auch auf Basis der dann neu fokussierten Dokumentation - gezielt die Einschätzung der Variablen trainiert werden.

Insbesondere in Bezug auf Vollzugslockerungen oder Entlassungen liefern die Prozessvariablen dahingehend einen Mehrwert, als dass sie kurzfristigere Veränderungen abbilden können, als dies die Ausprägung des umwelt- und personenbezogenen Problemprofils ermöglicht. So können v.a. die einzelnen Faktoren der Bereiche Wollen, Wissen und Können Entwicklungen beispielsweise innerhalb des letzten Jahres anschaulich darstellen. Dies ermöglicht es, Lockerungsentscheidungen standardisierter und nachvollziehbarer zu begründen.

Insgesamt scheinen die Variablen der Veränderungsmessung in Form von Veränderungsmotivation und Prozess- variablen eine sinnvolle, aussagekräftige und bereichernde Erweiterung im ROS-Prozess zu sein. Im Bereich der umweltbezogenen Messung besteht bezüglich einer reliablen Erfassungsmethode noch deutlicher Verbesserungsbedarf.

Des Weiteren stützen die Erkenntnisse den holistischen Ansatz aus personen- und umweltbezogener Arbeit mit den Straftäterinnen und Straftätern. Dies bestätigt einerseits das Konzept von ROS und kann andererseits ein Anstoß sein, die Auswirkungen der Bearbeitung umweltbezogener Aspekte vertieft zu betrachten und zu integrieren.

Ebenfalls kann festgehalten werden, dass die Rückfälligkeit seit Einführung von ROS deutlich zurückgegangen ist. Das ist insofern enorm wichtig, als dass ROS mit dem Ziel eingeführt wurde, Rückfälle zu vermeiden sowie eine nachhaltige Resozialisierung der verurteilten Person zu fördern. Die gewonnenen Erkenntnisse können also als Erfolg gewertet und gleichzeitig als Ziel genommen werden, ROS weiter zu optimieren.

Funding Open access funding provided by University of Zurich

Interessenkonflikt D. Treuthardt war u.a. an der Entwicklung und Einführung des ROS beteiligt. Außerdem ist D. Treuthardt Leiter der Bewährungs- und Vollzugsdienste des Kantons Zürich und der ROS-Administration, die die Konzeptverantwortung für ROS trägt. D. Treuthardt gibt an, dass dies keinen Interessenkonflikt darstellt, im Rahmen wissenschaftlicher Transparenz jedoch explizit offenzulegen ist. M. Kröger gibt an, dass kein Interessenkonflikt besteht.

Open Access Dieser Artikel wird unter der Creative Commons Namensnennung 4.0 International Lizenz veröffentlicht, welche die Nutzung, Vervielfältigung, Bearbeitung, Verbreitung und Wiedergabe in jeglichem Medium und Format erlaubt, sofern Sie den/die ursprünglichen Autor(en) und die Quelle ordnungsgemäß nennen, einen Link zur Creative Commons Lizenz beifügen und angeben, ob Änderungen vorgenommen wurden.

Die in diesem Artikel enthaltenen Bilder und sonstiges Drittmaterial unterliegen ebenfalls der genannten Creative Commons Lizenz, sofern sich aus der Abbildungslegende nichts anderes ergibt. Sofern das betreffende Material nicht unter der genannten Creative Commons Lizenz steht und die betreffende Handlung nicht nach gesetzlichen Vorschriften erlaubt ist, ist für die oben aufgeführten Weiterverwendungen des Materials die Einwilligung des jeweiligen Rechteinhabers einzuholen.

Weitere Details zur Lizenz entnehmen Sie bitte der Lizenzinformation auf http://creativecommons.org/licenses/by/4.0/deed.de.

\section{Literatur}

Andrews DA, Bonta J (2010) The psychology of criminal conduct. Routledge, London

Cohen J (1988) Statistical power analysis for the behavioral sciences, 2. Aufl. Erlbaum, Hillsdale

DiClemente CC, Hughes SO (1990) Stages of change profiles in outpatient alcoholism treatment. J Subst Abuse 2(2):217-235. https:// doi.org/10.1016/s0899-3289(05)80057-4

Hallgren KA (2012) Computing inter-rater reliability for observational data: an overview and tutorial. Tutor Quant Methods Psychol 8:23

Haubner-MacLean T, Heurix I, Eher R (2013) Violence Risk Scale (VRS) (Deutsche Übersetzung) 
Krippendorff K (2004) Reliability in Content Analysis: Some Common Misconceptions and Recommendations. Hum Commun Res 30(3):411-433. https://doi.org/10.1111/j.1468-2958.2004. tb00738.x

Loewe-Baur M (2017) Der Risikoorientierte Sanktionenvollzug (ROS). Ergebnisse und Erkenntnisse einer Evaluation. Dissertation, Universität Zürich

Prochaska JO, DiClemente CC (1984) The transtheoretical approach: crossing traditional boundaries of therapyboundaries of therapy. Dow Jones-Irwin, Homewood

Prochaska JO, DiClemente CC (1986) Toward a comprehensive model of change. In: Treating addictive behaviors. Springer, Boston, S 3-27

Ruflin R, Miani C, Dvorak A, Jörg R, Schnyder-Walser K (2013) Modellversuch Risikoorientierter Sanktionenvollzug. Schlussbericht Prozessevaluation. https://www.bj.admin.ch/dam/data/bj/ sicherheit/smv/modellversuche/evaluationsberichte/ros-schluss ber-eval-d.pdf. Zugegriffen: 10. Okt. 2019

$\mathrm{Saß} \mathrm{H}$, American Psychiatric Association (1998) Diagnostisches und statistisches Manual psychischer Störungen DSM-IV. Hogrefe,
Göttingen (übersetzt nach der vierten Auflage des Diagnostic and statistical manual of mental disorders der American Psychiatric Association)

Schwarzenegger C, Manzoni P, Baur M (2013) Modellversuch Risikoorientierter Sanktionenvollzug. Ergebnisevaluation Schlussbericht. https://www.bj.admin.ch/dam/data/bj/sicherheit/smv/ modellversuche/evaluationsberichte/ros-schlussber-ergebis-d. pdf. Zugegriffen: 10. Okt. 2019

Treuthardt D, Kröger M (2019) Der Risikoorientierte Sanktionenvollzug (ROS) - empirische Überprüfung des Fall-Screening-Tools (FaST). Schweiz Z Kriminol 19(1):76-85

Treuthardt D, Kröger M (2021) Evaluation des Risikoorientierten Sanktionenvollzugs - Konstruktion einer Veränderungsmessung. Forens Psychiatr Psychol Kriminol 15:73-83. https://doi.org/10. 1007/s11757-020-00641-w

Treuthardt D, Loewe-Baur M, Kröger M (2018) Der Risikoorientierte Sanktionenvollzug (ROS) - aktuelle Entwicklungen. Schweiz Z Kriminol 2018(2):24-32 